\title{
Interpersonal Entrainment in Dancers: Contrasting Timing and Haptic Cues
}

\section{George Sofianidis and Vassilia Hatzitaki}

\author{
Laboratory of Motor Control and Learning, Department of Physical Education and Sport \\ Sciences, Aristotle University of Thessaloniki, Thessaloniki, Greece
}

ABSTRACT: We examined the nature of interpersonal synchrony mediated by light touch when individuals rhythmically sway side by side at their own pace, same or different externally imposed tempos. Three types of couples participated in two experiments; dancer couples, non dancer couples, and mixed couples. Spectral analysis of the sway signals revealed that fingertip touch evoked interpersonal synchronization during self-paced sway. During metronome paced sway only dancers further increased synchronization suggesting a more efficient integration of tactile and timing cues. In a subsequent experiment, we asked whether dancers are able to suppress the spontaneous tendency towards entrainment when the two partners sway at different tempos. Touch evoked interpersonal entrainment only in mixed couples whereas touch interference was weaker in novices and absent in experts. All together, these results suggest that light fingertip touch evokes spontaneous interpersonal entrainment that is stronger when provided by a dancer who has developed the ability to modulate the self-organized properties of inter-personal entrainment.

KEYWORDS: Interpersonal coordination, light touch, dance expertise, rhythmic sway

\section{Introduction}

The self-organizing coordination dynamics that have been outlined in the HKB model for cyclical bimanual movements can also be depicted across two individuals performing rhythmic movements together while coupled by visual information. Moreover, it was shown that the interpersonal entrainment between two visually coupled partners can occur spontaneously and humans seem unable to avoid it, even if they are asked to do so.,
Spontaneous interpersonal synchrony has been demonstrated in humans performing other forms of rhythmic activity such as, rocking chairs together $^{5}$ and walking side by side ${ }^{6,7}$ or one in front of the other. ${ }^{8}$ The strength of spontaneous inter-personal coordination can be modulated by constraints imposed by the intrinsic Eigen-frequencies of the oscillating effectors, ${ }^{4-6}$ the sensory modality mediating contact ${ }^{6}$ and prior experience in rhythmic activities. ${ }^{9}$

Interpersonal entrainment has been observed when the partners involved in 
a rhythmic activity communicate by means of sensory feedback other than visual. Strong in-phase synchronization between individuals walking side by side $^{6}$ or one in front of the other ${ }^{8}$ was observed when increasing the mechanical coupling between partners. Moreover, visual and auditory communication resulted in less occurrence of gait synchronization. ${ }^{7}$ It should be noted however that in those studies neither the frequency, nor the amplitude of the movement was controlled during walking. Unintentional synchrony in this case may arise from the fact that partners were free to walk at their naturally preferred tempo.

In a series of studies that have been published elsewhere ${ }^{1,2}$ we investigated the effects of haptic information coupling and frequency constraints on the inter-personal coordination dynamics of voluntary rhythmic side-by-side sway.An additional motivation was to investigate the effects of traditional dance expertise on the inter-personal synchrony. In this paper, after providing a brief overview of the experimental methods and results, we attempt to discuss the overall combined implications of the two studies.

\section{Methods}

\subsection{Participants}

One hundred fourteen young volunteers participated in two experiments divided in three types of groups and tested in couples; expert couples, consisting of individuals with at least 8 years systematic practice in traditional Greek dance; novice couples, consisting of individuals with no prior experience in dance and mixed couples, consisting of one expert dancer and one novice partner. All participants were free from any neurological or musculoskeletal impairment and gave their informed consent prior to their inclusion in the study. For further details on the cohort characteristics refer to the previous papers. ${ }^{1,2}$

\subsection{Task and Procedure}

Experiment 1 required participants to stand in pairs on two adjacent force platforms while facing forward and perform a 40 second voluntary rhythmic sway task in the sagittal plane with eyes closed a) at a self-selected comfortable frequency (Self-Paced, SP sway) and b) at a pre-determined frequency of 0.25 $\mathrm{Hz}$ that was commonly set for the two partners by a metronome signal (Metronome-Paced, MP sway). The task was performed either with Haptic Contact (HC) or no contact (NC) resulting in four experimental conditions (Figure 1).

In experiment 2, partners stood in the same position but they performed a 60 second voluntary rhythmic sway task which was differently paced between the two partners by a metronome signal (slow frequency: $0.25 \mathrm{~Hz}$, fast frequency: $0.35 \mathrm{~Hz}$ ). The sway task was performed under two experimental conditions (Figure 2): 1) with Haptic Contact established in the 2 nd trial segment ( $\mathrm{NC} \_\mathrm{HC}$ ),

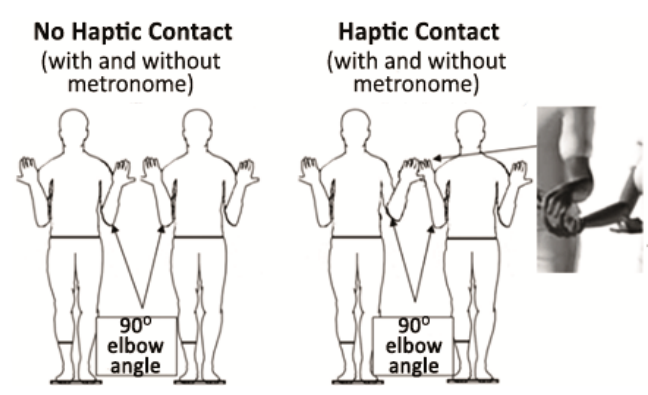

Figure 1: Partners' position in experiment 1. 


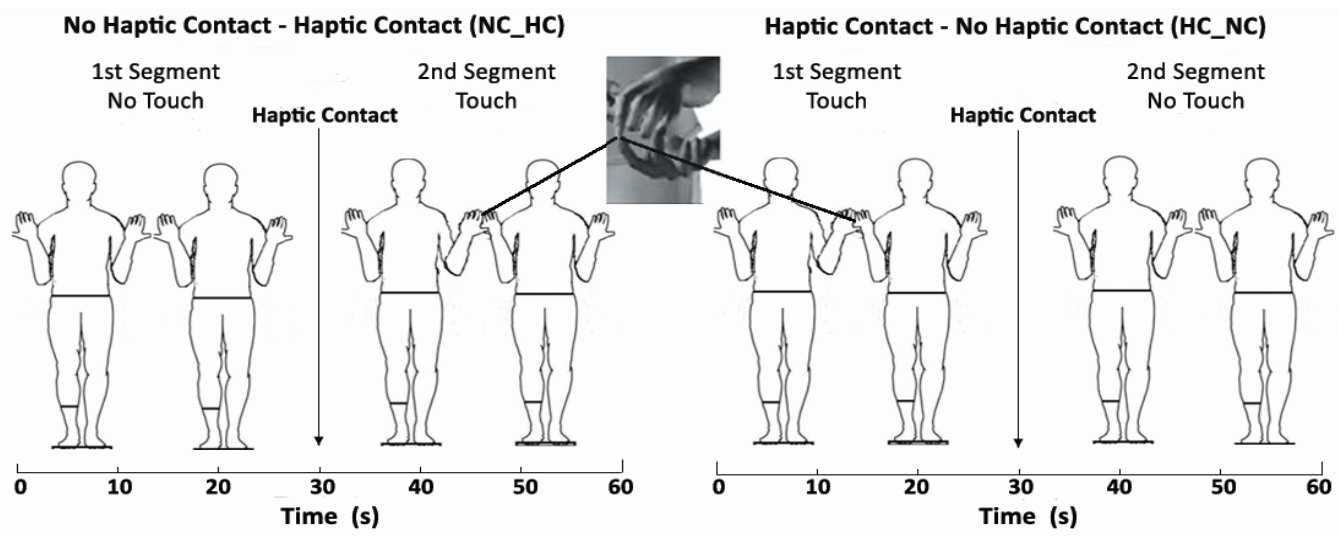

Figure 2: Experimental task during the two Haptic Contact conditions in experiment 2.

2) with Haptic Contact released in the $2^{\text {nd }}$ trial segment (NC_HC).

\subsection{Data Reduction and Analysis}

In experiment 1 the degree of synchronization between the partners' sways in the frequency domain was assessed using cross-spectral analysis to determine the coherence and phase spectra of the two Centre of Pressure (CoP) signals ${ }^{4,5}$. The coherence (nu) and cross-spectral estimate of RP $\left({ }^{\circ}\right)$ were determined at the dominant sway frequency from the coherence and phase spectrum respectively. For MP sway, the dominant sway frequency was set by the metronome at $0.25 \mathrm{~Hz}$. The distribution of the crossspectral RP angles across the five sway trials performed in each condition was determined. These distributions consisted of nine $20^{\circ} \mathrm{RP}$ regions between $0^{\circ}$ and $180^{\circ}$ and were used to calculate the frequency (percentage) of occurrence in each of the nine RP regions for each condition. ${ }^{4,5}$

Similarly, in experiment 2 the effect of touch on the partners' sway was examined using spectral analysis in order to determine the frequency characteris- tics of the CoP displacement signal. From each CoP signal the deviation of the dominant from the target frequency was calculated. In addition, coherence analysis was used to assess the strength of the relationship between the partners' CoP signals (inter-personal coherence) and between the $1^{\text {st }}$ and $2^{\text {nd }}$ trial segment of each partner's CoP signal (intra-personal coherence) within the trial.

\subsection{Statistical Analysis}

In experiment 1, the effect of group, haptic contact, and metronome on each spectral measure was evaluated employing a 3 (Group) - 2 (Condition) - 2 (Metronome) ANOVA with repeated measures on condition and metronome. Moreover, the distribution of the RP angles across the nine phase regions was submitted to a 3 (Group) - 2 (Condition) - 2 (Metronome) - 9 (RP region) repeated measures ANOVA.

In experiment 2, the effect of the Group, Trial Segment, Contact Condition and Frequency on each spectral measure was evaluated using a 3(Group) $x$ 2(Trial segment) $x 3$ (Condition) $x$ 
2(Frequency) ANOVA with repeated measures on Trial Segment, Condition and Frequency. The inter-personal coherence measure was submitted to a 3(Group) $x$ 2(Trial segment) $x$ 3 (Condition) ANOVA run separately at the 0.25 and $0.35 \mathrm{~Hz}$ target frequencies. The intra-personal coherence measure was submitted to a 3(Group) $x$ 2(Partner) $\times$ 2(Condition) repeated measures ANOVA that was run separately at the 0.25 and $0.35 \mathrm{~Hz}$ target frequency. Significance level was set at $p<0.05$.

\section{Results}

\subsection{Experiment 1}

The magnitude of coherence between the partners' CoP signals significantly increased with haptic contact $(F(1,27)=$ $257.07, p<0.001)$ and metronome guidance $(F(1,27)=54.74, p<0.001)$. Moreover a significant condition by metronome interaction $F(1,27)=225.31$, $p<$ 0.001 ,suggests that haptic contact did not further improve the degree of coherence between the partners' CoP signals during MP sway (figure 3).However, this effect was dependent on the group $F(2,27)=3.968, p<0.05$. A post hoc paired samples comparisons revealed that when sway was paced by the metronome, only the expert dancers significantly increased the magnitude of coherence with haptic contact, $t(9)=-$ 3.595, $p<0.01$.

The relative phase distribution analysis revealed a significant main effect of the RP Region, $[F(2.007,48.17)=66.44$, $p<0.001]$, as well as a significant Condition by RP Region interaction, [F(4.37, 104.81) $=68.39, p<0.001]$, suggesting that with haptic contact the percent oc-

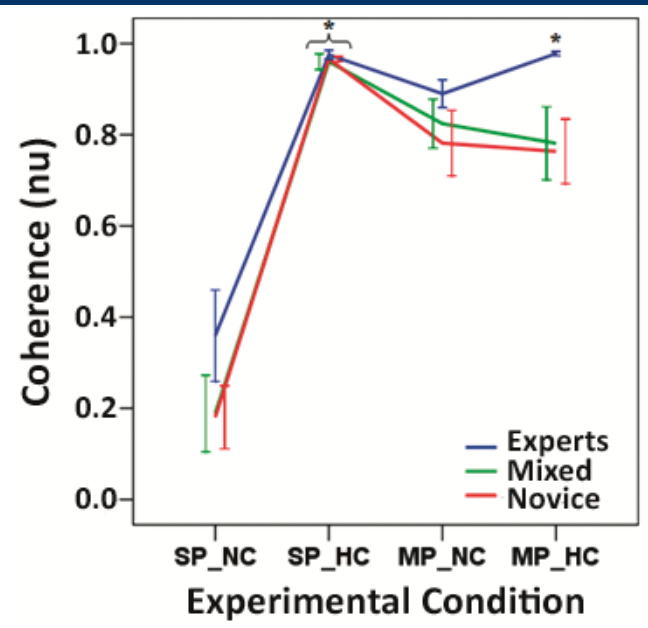

Figure 3: Magnitude of coherence between the partners' sway signals plotted across the sway conditions (self-paced with no contact (SP_NC), self-paced with haptic contact (SP_HC), metronome-paced with no contact (MP_NC) and metronome-paced with haptic contact (MP_HC) separately for each group. Group means \pm 1 SD. *: Significantly different than performance with no contact $(p<0.001)$.

currence of RP angles increased significantly in the $0^{\circ}-20^{\circ}$ phase region, while in no contact condition RP values were evenly distributed across all nine regions (Figure 4). Furthermore, a significant Condition - Metronome by RP Region interaction, $[F(3.58,85.85)=27.51, p<$ $0.001]$, suggests that the effect of haptic contact on the concentration of the RP angles was dependent on the pacing stimulus. Post hoc comparisons across the phase regions performed separately for SP and MP sway revealed that for SP sway the percent of RP angles significantly increased with haptic contact in the $0^{\circ}-20^{\circ}$ region. In MP sway on the other hand, only the expert dancers significantly increased the concentration of $\mathrm{RP}$ angles in the $0^{\circ}-20^{\circ}$ region with haptic contact (Figure 4). 


\section{Experimental Condition}

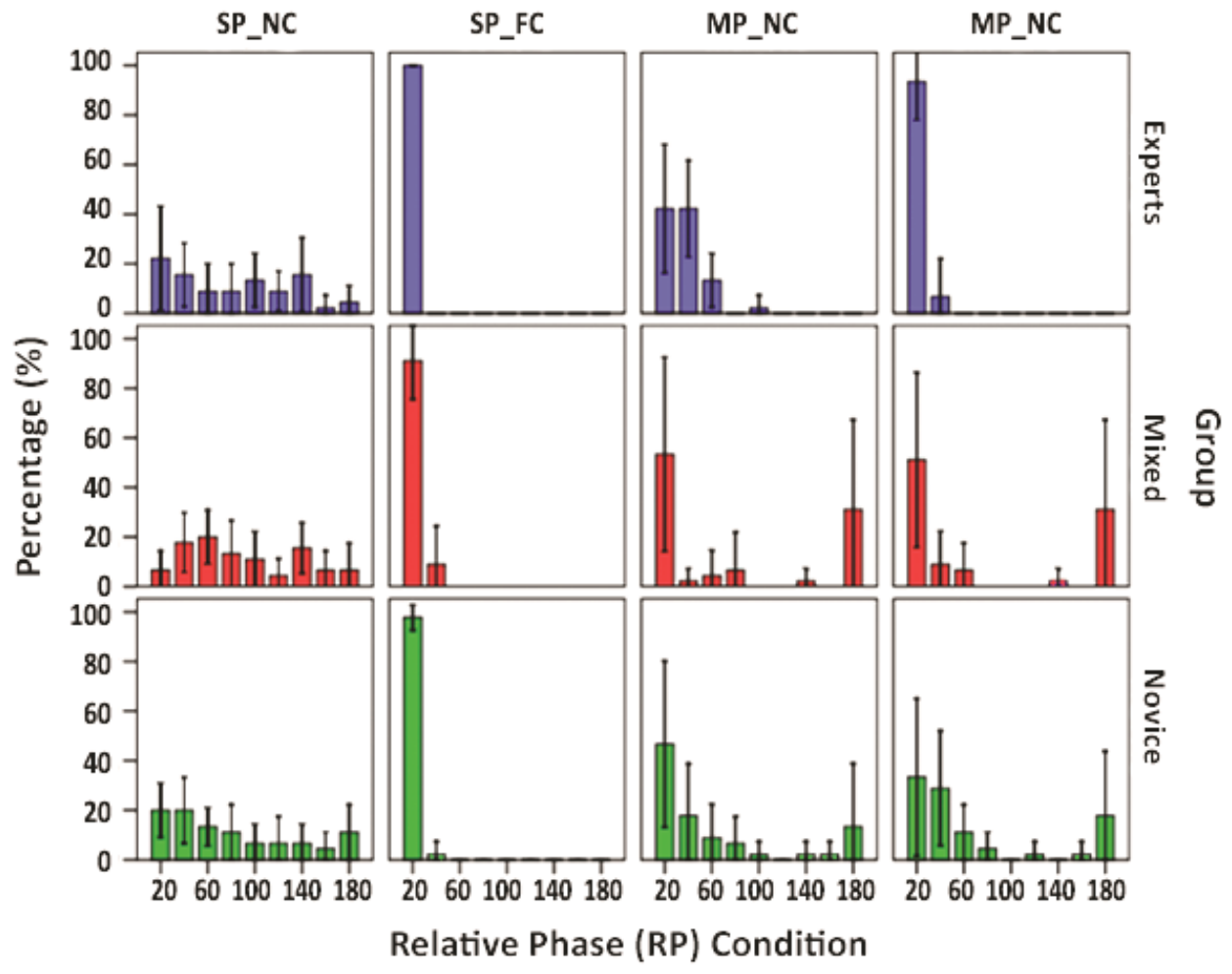

Figure 1: Distribution of the relative phase (RP) angles across the nine phase regions plotted across the four sway conditions separately for each group. Group means \pm 1 SD.

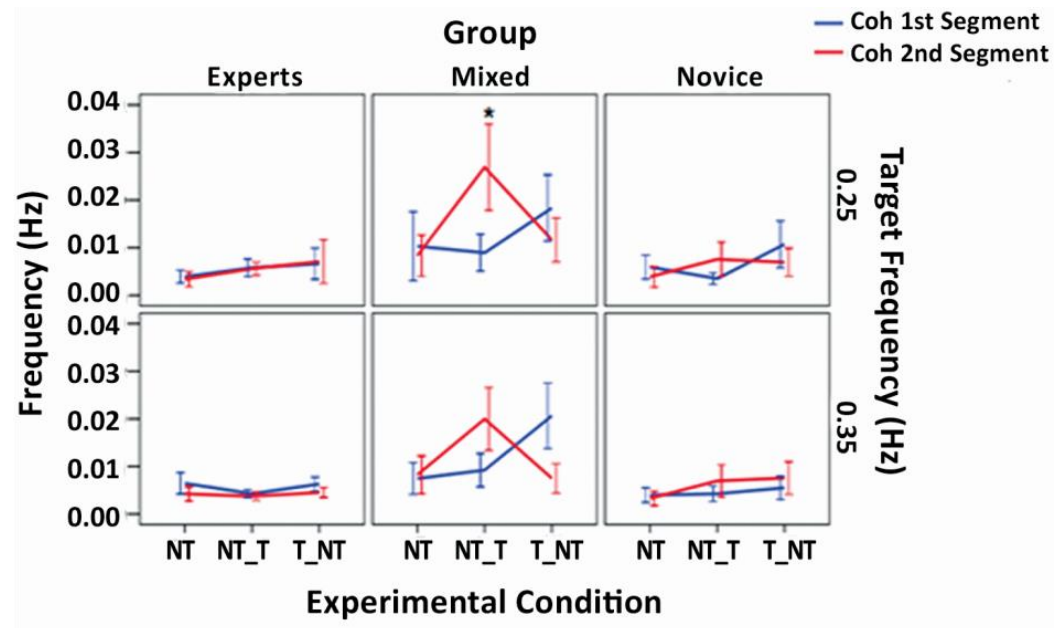

Figure 5: Deviation of the dominant from the target sway frequency

*: significantly different than 1 st segment at $p<0.05$. 


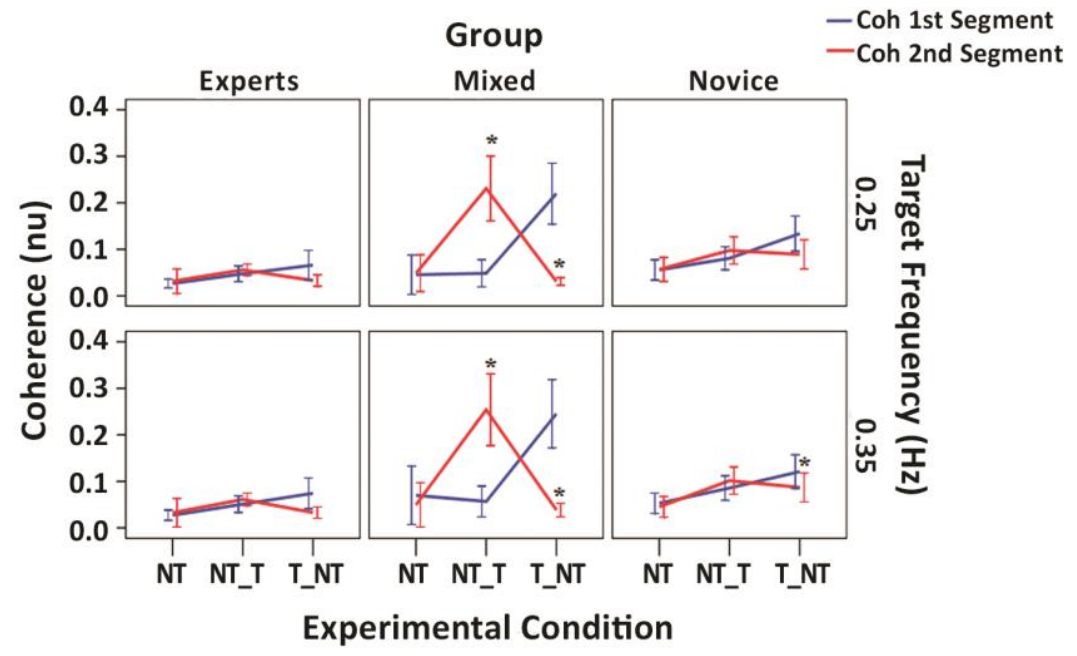

Figure 6: Inter-personal coherence (between partners) at the target frequency. *: significantly different than 1 st segment at $p<0.05$.

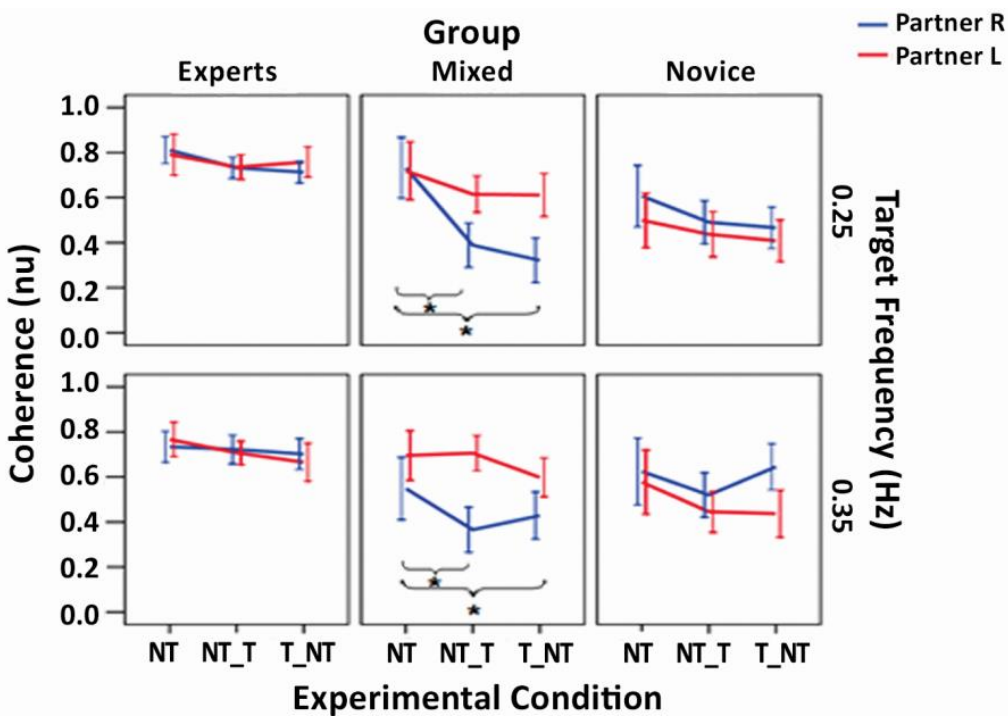

Figure 7: Intrapersonal coherence (between the 1st and 2nd trial segment) for the Right and Left standing partner. In the Mixed group, the Right standing partner is the novice. *: significantly different than NT at $p<0.05$.

\subsection{Experiment 2}

Analysis revealed that haptic contact increased the deviation of the dominant from the target sway frequency (Figure $5)$, decreased the proportion of the sig- nal's power at the target frequency, increased the coherence between the partner's CoP sway signals (Figure 6) and also increased the coherence between the $1^{\text {st }}$ and $2^{\text {nd }}$ trial segment (Figure 7). However these effects were specific to 
the mixed group partners whereas haptic interference was weaker in the novices and absent in experts. For a full description of the results refer to the previously published paper. ${ }^{1,2}$

\section{Discussion}

The main findings of the two studies are summarized as follows: a)interpersonal light touch evokes a strong unintentional sway synchrony regardless of whether sway is self or metronome paced, b) when sway is constrained by a metronome only expert dancers can benefit from haptic contact to further improve interpersonal synchrony, c) when sway is differently paced between partners, dancers maintain their synchronization to the metronome without interference from haptic contact with their partner, d) novices on the other hand, show evidence of interpersonal entrainment with haptic contact and finally e) interpersonal entrainment is the greatest in the mixed couples where the novice is attracted to the sway frequency of his/her expert partner with contact. These findings are discussed within the frameworks of dynamical systems and sensory integration theories.

In first experiment we have shown that interpersonal tactile feedback (light touch $<1 \mathrm{~N})^{10}$ induces a strong spontaneous synchronization between the individual periodic sways. This finding complements those of previous studies showing that sensory feedback coupling (visual or auditory) increases unintentional synchronization between individuals involved in a rhythmic activity. This relationship may be well captured by the self-organizing dynamics of coupled oscillators that have described in previous studies. ${ }^{4,11}$ However, tactile feed- back seems to be a stronger (coherence magnitude close to 0.95) medium of spontaneous interpersonal synchrony when compared to visual coupling (coherence magnitude close to 0.6) between partners swaying wrist pendulums $^{4,11}$ or rocking their chairs together. ${ }^{5}$ The stronger effect of tactile feedback when compared to either visual or auditory information exchanged between partners has been also confirmed in side by side walking. ${ }^{6}$ This could be due to the nature of the feedback signal provided by the different sensory modalities. Haptic cues seem to be a less noisy source of movement information compared to visual cues. In this case, we assume that the body acts as a single pendulum (lower and upper body in-phase coupling) so that individuals can sense the other partner's sway through touch and adopt his/her sway motion in response to sensory cues from the other partner.

When sway frequency was paced by the metronome $(0.25 \mathrm{~Hz})$, only expert dancers were able to further improve spontaneous interpersonal synchrony with haptic contact while mixed and novice couples show a more relative form of coordination. The absence of a strong in-phase attractor state with haptic contact in novice and mixed couples under conditions of metronome paced sway could be due to the fact that nonexperienced performers deal with the pacing stimulus as an additional constraint. Periodic swaying at a pace imposed by a metronome requires the matching of the sway cadence to the metronome pace in addition to the effort of maintaining haptic contact with the other partner. This assumes the presence of a multimodal sensory inte- 
gration process which is parameterized by the rate and context in which touch and sound are bound into a single, coherent unit. $^{12}$ Traditional dancers, through long-term practice, have learned to integrate sensory stimuli from visual, tactile and auditory sources into their rhythmical sway pattern.

In the $2^{\text {nd }}$ experiment however, touch and sound were not coherent but provided conflicting information about sway. Our results showed that expert dancers have the capacity to segregate the two stimuli, prioritizing the synchronization to the metronome signal while "ignoring" the haptic signal. The prioritization of the metronome to the haptic cue could be explained by the nature and physical characteristics of the two signals. Individuals tend to be more stable and closer to the instructed coordination when coordinating their movements with periodic discrete auditory rather than continuous visual cues. ${ }^{13}$ Assuming that dancers' time perception and sensory-motor synchronization skill are modulated by their prior experience in dancing, it seems reasonable why dancers were more entrained to the periodic discrete stimuli of the metronome rather than the more unreliable continuous touch stimulus.

Interpersonal entrainment due to haptic contact was greatest in the mixed couples. Interestingly, the novice partner's sway was consistently attracted toward the sway frequency of the expert dancer suggesting that the expert was leading the novice partner. This directional effect on touch interpersonal interference could be due to more reliable sway feedback signal the novice partner receives at the haptic contact. ${ }^{12}$ Performance stability and its associated low motor output variability is a well-established feature of motor expertise ${ }^{14}$ and of dancers in particular. ${ }^{15}$ It seems that the novice partner is easily entrained to the expert's more stable sway signal perceived through haptic contact than he/she would be in the case of perceiving a more variable feedback signal about sway.

In conclusion, the results of the two experiments described in this paper support the existence of expert-novice differences in the multimodal sensory integration of touch and sound underlying the voluntary control of rhythmic actions and the spontaneous emergence of haptic mediated interpersonal entrainment. Based on the current evidence it is argued that systematic practice in dance could differently shape the central nervous system possibly by modulating its self-organizing properties as well.

\section{Acknowledgements}

The research performed in this project has received funding from the European Union (European Social Fund - ESF) and Greek national funds through the Operational Program "Education and Lifelong Learning" of the National Strategic Reference Framework (NSRF) - Research Funding Program: Heracleitus II. Investing in knowledge society through the European Social Fund.

\section{References}

1. Sofianidis G, Hatzitaki V, Grouios G, Johannsen L, Wing A. Somatosensory driven interpersonal synchrony during rhythmic sway. Hum Mov Sci. 2012 Jun;31(3):553-66. doi: 10.1016/j.humov. 2011.07.007. 
2. Sofianidis G, Elliott MT, Wing AM, Hatzitaki V. Can dancers suppress the haptically mediated interpersonal entrainment during rhythmic sway? Acta Psychol (Amst). 2014Jul;150:106-13. doi: 10.1016/j.actpsy.2014.05.002.

3. Schmidt RC, Carello C, Turvey MT. Phase-Transitions and Critical Fluctuations in the Visual Coordination of Rhythmic Movements between People. J Exp Psychol Hum Percept Perform. 1990 May;16(2):227-47. doi: 10.1037/00961523.16.2.227.

4. Schmidt RC, O'Brien B. Evaluating the dynamics of unintended interpersonal coordination. Ecol Psychol. 1997 Jan;9(3):189-206. doi: 10.1207/s1532 6969eco0903_2.

5. Richardson MJ, Marsh KL, Isenhower RW, Goodman JRL, Schmidt RC. Rocking together: Dynamics of intentional and unintentional interpersonal coordination. Hum Mov Sci. 2007 Dec;26(6):86791. doi: 10.1016/j.humov.2007.07.002.

6. Nessler JA, Gilliland SJ. Interpersonal synchronization during side by side treadmill walking is influenced by leg length differential and altered sensory feedback. Hum Mov Sci. 2009 Dec;28(6):772-85. doi: 10.1016/j.humov. 2007.07.002.

7. Zivotofsky AZ, Hausdorff JM. The sensory feedback mechanisms enabling couples to walk synchronously: an initial investigation. J Neuroeng Rehabil. 2007 Aug 8;4:28. doi: 10.1186/1743-0003-428.

8. Harrison SJ, Richardson MJ. Horsing around: spontaneous four-legged coordination. J Mot Behav. 2009 Nov;41(6): 519-24. doi:10.3200/35-08-014.
9. Issartel J, Marin L, Cadopi M. Unintended interpersonal co-ordination: "we march to the beat of our own drum?". Neurosci Lett. 2007 Jan 16; 411(3):174-9. doi: 10.1016/j.neulet. 2006.09.086.

10. Johannsen L, Guzman-Garcia A, Wing AM. Interpersonal Light Touch Assists Balance in the Elderly. J Mot Behav. 2009 Oct;41(5):397-9. doi: 10.3200/35-09-001.

11. Richardson MJ, Marsh KL, Schmidt RC. Effects of visual and verbal interaction on unintentional interpersonal coordination.J Exp Psychol Hum Percept Perform. 2005 Feb;31(1):62-79. doi: 10.1037/0096-1523.31.1.62.

12. Elliott MT, Wing AM, Welchman AE. Multisensory cues improve sensorimotor synchronisation. Eur J Neurosci. 2010 May;31(10):1828-35. doi: 10.1111/j.14 60-9568.2010.07205.x.

13. Varlet $M$, Marin L, Issartel J, Schmidt RC, Bardy BG. Continuity of Visual and Auditory Rhythms Influences Sensorimotor Coordination. PLoS One. 2012;7(9):e44082. doi: 10.1371/journal. pone. 0044082 .

14. Cohen RG, Sternad D. Variability in motor learning: relocating, channeling and reducing noise. Exp Brain Res. 2009 Feb;193(1):69-83. doi: 10.1007/s00221008-1596-1.

15. Golomer E, Cremieux J, Dupui P, Isableu B, Ohlmann T. Visual contribution to self-induced body sway frequencies and visual perception of male professional dancers. Neurosci Lett. 1999 Jun 4;267(3):189-92. doi: 10.1016/ S0304-3940(99)00356-0. 